\title{
Nonoxidized Al-overcoated Ir bilayers with high reflectance in the extreme ultraviolet above $50 \mathrm{~nm}$
}

\author{
Juan I. Larruquert \\ José A. Méndez \\ José A. Aznárez \\ Instituto de Física Aplicada-CSIC \\ C/Serrano 144 \\ 28006-Madrid, Spain \\ E-mail: larruquert@io.csic.es
}

\begin{abstract}
Bilayers of a thin, nonoxidized Al film over an Ir film are prepared. The bilayers are intended to enhance the reflectance of Al below the Al plasma wavelength $\left(\lambda_{p} \sim 83 \mathrm{~nm}\right)$. Above $\lambda_{p}$ the bilayers have a high reflectance comparable to that of single Al films. Ir films were deposited by electron gun evaporation on a heated substrate. Al films of various thicknesses are deposited by evaporation from a thermal source on the Ir film once it is cooled to room temperature. The bilayers are prepared under ultra-high-vacuum (UHV) conditions and their reflectance is measured in situ. Accelerated aging processes, which are performed by annealing the bilayers in UHV, show that the bilayers are stable after $1 \mathrm{~h}$ annealing at temperatures up to $540 \pm 25 \mathrm{~K}$. The bilayers show no observable deterioration after long-term storage under normal atmosphere. The optical constants of Ir films are also obtained. In-orbit coating of optical surfaces with Ir/AI bilayers is proposed for extreme UV astronomy. () 2002 Society of Photo-Optical Instrumentation Engineers. [DOI: $10.1117 / 1.1477438]$
\end{abstract}

Subject terms: extreme ultraviolet; far ultraviolet; multilayers; reflectance; space optics; thin films, optical properties; iridium; aluminum.

Paper 010143 received Apr. 25, 2001; revised manuscript received Dec. 12, 2001; accepted for publication Dec. 14, 2001.

\section{Introduction}

The spectral region below $125 \mathrm{~nm}$, which is referred to here as the extreme ultraviolet (EUV), contains a wealth of spectral lines important to astrophysics, solar physics, and atmospheric physics. The low intensity of many EUV radiation sources in the universe requires the development of new coatings with higher EUV normal reflectance and/or spectral selectivity than those available today. The standard coatings for wavelengths above $85 \mathrm{~nm}$, such as ion-beamdeposited $\mathrm{SiC}$ and $\mathrm{B}_{4} \mathrm{C}$, have moderate normal reflectance of $\sim 0.3$. This value is low for many applications where either low-intensity sources and/or optical instruments with several reflections are involved. Only nonoxidized Al films are known to provide a high reflectance that may reach 0.9 above $90 \mathrm{~nm}$. The use of nonoxidized Al coatings are currently a technological challenge, due to the high reactivity of $\mathrm{Al}$ in presence of oxygen and water vapor. As a consequence, an aluminum oxide layer immediately grows on the Al film, resulting in a dramatic decay of the $\mathrm{Al}$ reflectance for wavelengths below $200 \mathrm{~nm}$.

An ancient proposal by Hass and Hunter ${ }^{1}$ suggested a procedure to take advantage of the high reflectance of $\mathrm{Al}$ films for astronomical observations in the EUV: the overcoating of the optics with Al would be performed once the instrument would be placed in a high enough orbit. Thus, the natural vacuum present in the orbit would preserve the Al films from oxidation during a time compatible with the mission. In this way, the reflectance of conventional coatings could be exceeded by a factor of 3 at every reflection.
Below the Al plasma wavelength $\left(\lambda_{p} \sim 83 \mathrm{~nm}\right)$, the reflectance of Al films abruptly decreases to very low values in a transition where Al turns into a low absorbing material. However, there are spectral lines below $\lambda_{p}$ that are important for astrophysics, of species such as $\mathrm{H}_{2}$, HeI, OII to OV, CII, NII to NIV, NeI, MgII, SiII, SII to SV, FeIII, etc. Hass and Hunter ${ }^{1}$ proposed a way to extend the high reflectance of Al toward shorter wavelengths by overcoating an Ir film with a thin Al film. Such Ir/Al bilayers would combine the high reflectance of $\mathrm{Al}$ above $\sim 83 \mathrm{~nm}$ with the moderate reflectance of the underlying Ir film, taking advantage of the low absorption of Al below $\lambda_{p}$. Calculations of the bilayer reflectance were presented in Ref. 1, although no experimental work was performed.

The in-orbit Al coating application has fascinated the scientific community for decades but it is still a technological challenge. Some advances have been performed toward the development of this technology. The European Space Agency's project EDMO (Experiment for the Deposition of Materials in Orbit) tested the space performance of thermal evaporation sources by the deposition of thin films of $\mathrm{Al}$ and other materials under microgravity. ${ }^{2}$ Also, an extensive laboratory research has been performed ${ }^{3-8}$ at the Spanish Consejo Superior de Investigaciones Científicas on nonoxidized Al coatings for the EUV. However, more advances are still necessary in several fields before the in-orbit coating application can be implemented, such as in-orbit substrate cleaning, masking of the sensitive optical surfaces (such as cameras) to prevent $\mathrm{Al}$ condensation in these areas, and the design of space multiple evaporation sources for 


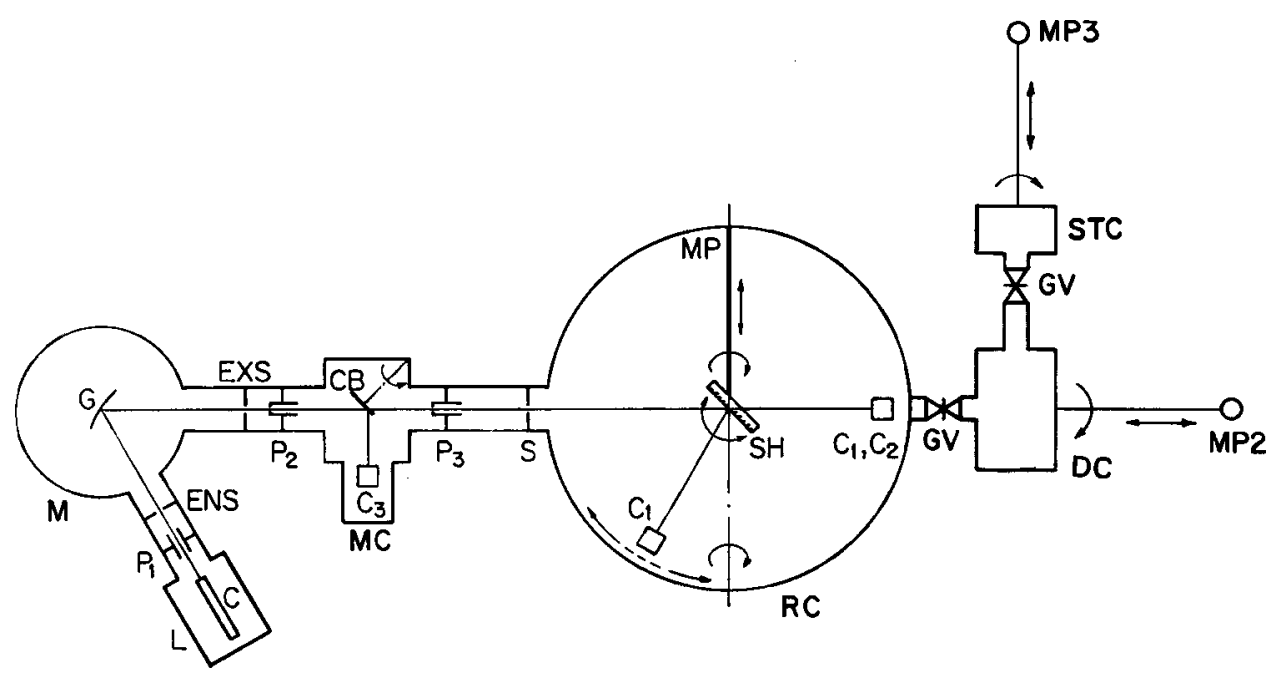

Fig. 1 Schematic view of the experimental equipment: C, capillary; L, lamp; M, monochromator; G, diffraction grating; ENS, entrance slit; EXS, exit slit; MC, modulation chamber; CB, chopper blade; S, aperture; $\mathrm{RC}$, reflectance chamber; $\mathrm{C}_{1}, \mathrm{C}_{2}$, and $\mathrm{C}_{3}$, channel electron multipliers; MP, MP2, and MP3, sample manipulators; $\mathrm{SH}$, sample holder; $\mathrm{P}_{1}, \mathrm{P}_{2}$, and $\mathrm{P}_{3}$, small conductance pipes; $\mathrm{DC}$, deposition chamber; STC, sample transfer chamber; and GV, gate valve.

in-orbit coating of large surfaces. The benefits of such a technology would not be limited to the much higher reflectance of the optical coatings, but would also reduce the problem of coating aging during optics storage in the period from deposition to in-orbit operation.

In this paper, we present experimental work on bilayers composed of a thin Al film over an Ir film and analyze their enhanced EUV reflectance at wavelengths below $\lambda_{p}$ of Al. The stability of the bilayers was investigated through annealing processes in vacuum and long-term storage under normal atmosphere. The optical constants of Ir films deposited both at room temperature as well as on hot substrates are also shown.

\section{Experimental Techniques}

The preparation of the multilayer coatings and the EUV reflectance measurements were performed in a system for thin film deposition and reflectometry described elsewhere. ${ }^{9,10}$ The system consists of several interconnected stainless steel chambers provided with individual pumping systems. Figure 1 shows a sketch of the chambers and main instruments. The system can be divided into two sections: one for the radiation propagation and the other for thin film deposition and treatments and in/out sample transfer. A new substrate is loaded in the sample transfer chamber (STC) and then it is transferred to the deposition chamber (DC) for substrate coating by means of a long linear/ rotary feedthrough (MP3). Two evaporation systems were implemented in the DC to deposit multilayers. Another long linear/rotary feedthrough (MP2) transfers the freshly coated substrate to the reflectance measurement chamber (RC) for reflectance and/or transmittance measurements without exposing it to atmosphere. Radiation propagates from the lamp (L) to the $\mathrm{RC}$, through the monochromator (M) and the modulation chamber (MC). Reflectance can be measured from near normal to near grazing incidence in two perpendicular planes of incidence: the horizontal and the vertical plane. DC and RC are under ultrahigh vacuum (UHV).

We evaporated $99.9 \%$ pure Ir by means of a $6-\mathrm{kW}$ electron gun. Ir was deposited at a rate of $\sim 0.01 \mathrm{~nm} / \mathrm{s}$ on both unheated and heated float glass substrates. When evaporating at larger deposition rates Ir particles were ejected from the melted Ir mass. For that reason, the preceding deposition rate was not exceeded. A heating device provided with a thermocouple placed in contact with the substrate and with a tungsten filament placed between the sample and the electron gun was used to heat the substrate at $540 \pm 25 \mathrm{~K}$ during Ir deposition. Ir films deposited on hot substrates resulted in higher EUV reflectance compared with films deposited on room temperature substrates, in agreement with the literature. ${ }^{11}$ Ir films deposited on heated substrates were later overcoated with an Al film. The Ir film was allowed to cool to room temperature before being overcoated with $\mathrm{Al}$. The $99.999 \%$ pure $\mathrm{Al}$ was evaporated using a tungsten filament. The $\mathrm{Al}$ deposition rate ranged from 0.5 to 1.0 $\mathrm{nm} / \mathrm{s}$.

The base pressure after bakeout at $470 \mathrm{~K}$ was 3 $\times 10^{-8} \mathrm{~Pa}$ in the DC and $10^{-8} \mathrm{~Pa}$ in the RC. The deposition of $\mathrm{Al}$ and $\mathrm{Ir}$ films was performed at a pressure ranging from $10^{-6}$ to $10^{-5} \mathrm{~Pa}$ and 1 to $5 \times 10^{-5} \mathrm{~Pa}$, respectively. Reflectance measurements were always performed on samples at room temperature. The film thickness was measured in situ by means of a quartz crystal monitor, which was calibrated through Tolanski interferometry.

\section{Experimental Results and Discussion}

\subsection{Reflectance and Stability of Ir/Al Bilayers}

Bilayer coatings with different Al film thickness on an Ir film were prepared. Figure 2 shows the experimental reflectance of a 15- and a 30-nm-thick Al film on a 20-nm-thick Ir film. The Ir films were deposited on glass substrates 


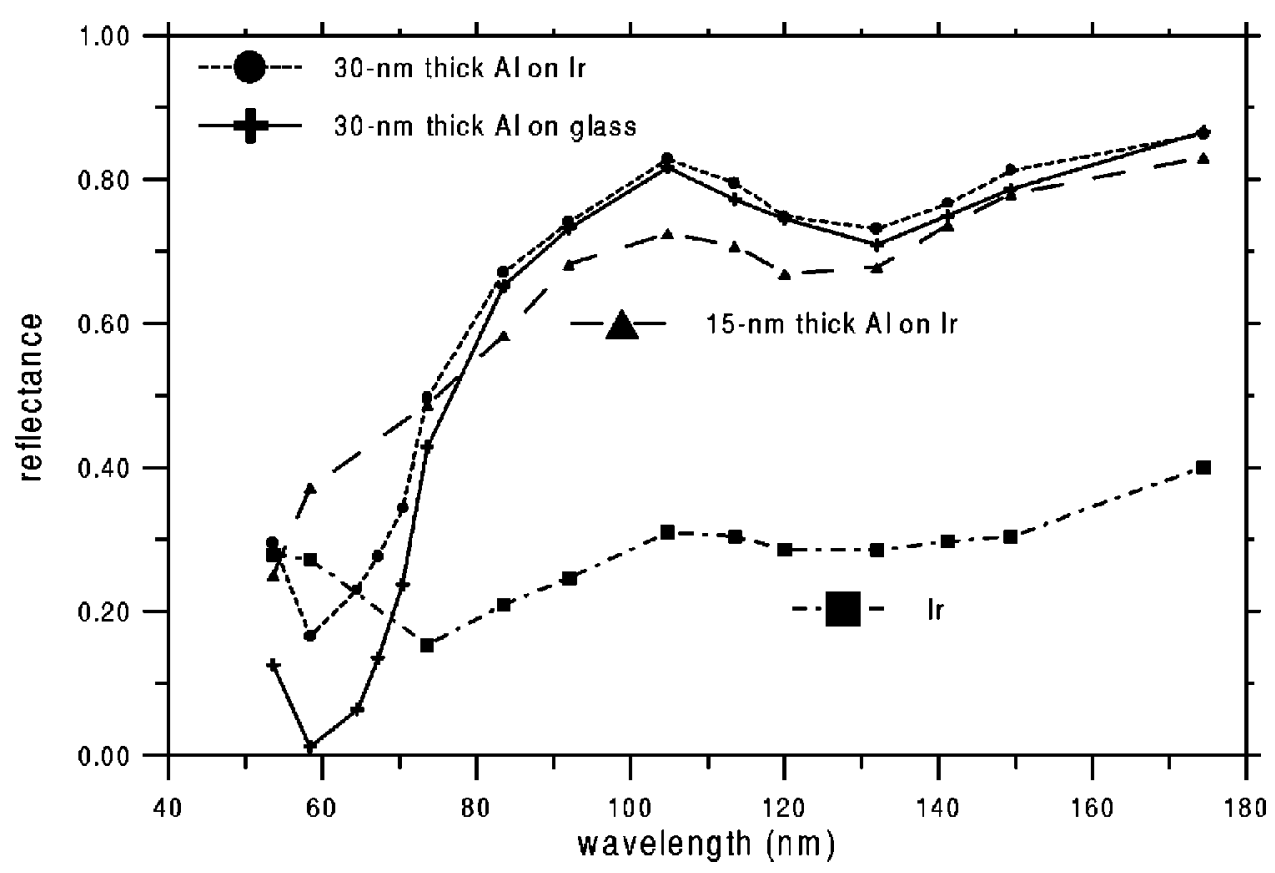

Fig. 2 Reflectance of a 15- and a 30-nm-thick Al film on a 20-nm-thick Ir film. Reflectance of a 30 -nm-thick Al film on glass and of a 20 -nm-thick Ir film on glass are also plotted.

heated at $540 \pm 25 \mathrm{~K}$. The Ir film for the sample with a 30-nm-thick Al film did not coat the whole surface of the glass substrate, whereas the Al film coated the whole surface. Then, the reflectance could be measured in the two different sample areas: one area with the Al film over the Ir film, and the other area with the Al film over the glass substrate. Reflectance on the latter area is also represented in Fig. 2. The reflectance on the 20-nm-thick Ir film, measured before overcoating it with $\mathrm{Al}$, is also shown. The reflectance of the Al film both on the Ir film and on the glass substrate are very close at wavelengths above $\lambda_{p}$ of Al. This is due to the increasing reflectance and absorption of $\mathrm{Al}$ for wavelengths longer than $\lambda_{p}$, which strongly reduce the radiation intensity reaching the underlying material. The Al-coated Ir film shows a higher reflectance than a single $\mathrm{Al}$ film below $\lambda_{p}$ of $\mathrm{Al}$, as desired. The $\mathrm{Ir} / \mathrm{Al}$ bilayer with a 15-nm-thick Al film exhibits a higher reflectance than the bilayer with a 30-nm-thick film at wavelengths of $73.6 \mathrm{~nm}$ and below, whereas reflectance of the latter bilayer is higher at $82.6 \mathrm{~nm}$ and above. Hence, a thinner $\mathrm{Al}$ film on Ir results in a higher reflectance enhancement in the spectral region of low absorption of Al. However, it also results in a lower reflectance above $\lambda_{p}$ because the thin Al film is not as efficient as an opaque film. Therefore, the optimum Al thickness will be a compromise of acceptable reflectance at both spectral regions and will depend on the practical application. The reflectance of the Al-coated Ir below $\lambda_{p}$ of $\mathrm{Al}$ is not only higher than the reflectance of pure $\mathrm{Al}$, but it is also higher than the reflectance of uncoated Ir for wavelengths down to $\sim 55 \mathrm{~nm}(\sim 64 \mathrm{~nm})$ for the $15-\mathrm{nm}-(30$ nm)-thick Al-overcoated Ir. Summarizing, Al-overcoated Ir is a highly efficient coating that combines both the high reflectance of $\mathrm{Al}$ above $\lambda_{p}$ with the moderate reflectance of Ir below $\lambda_{p}$.
A reflectance dip centered at 120.0 to $132.0 \mathrm{~nm}$ is observed in Fig. 2. The dip is attributed to surface plasmon excitation in the Al film. Surface plasmons are excited by interaction of the radiation impinging on the film with surface roughness components that have spatial wavelengths (the inverse of spatial frequencies) of the order of or shorter than the radiation wavelength. Since the reflectance dip is identical both for $\mathrm{Al}$ on $\mathrm{Ir}$ and for $\mathrm{Al}$ on glass, the dip is independent of the underlying Ir film. The use of superpolished substrates might result in a reflectance dip reduction.

A study by Herzig and Spencer ${ }^{12}$ showed that Ir/Al bilayers were unstable over time. To analyze the stability of the Ir/Al interface we carried out several aging tests. We performed annealing processes on various samples to accelerate sample aging. The aging studies on the Ir/Al bilayer reflectance cannot be performed under atmosphere because of the dramatic reflectance decrease caused by the Al oxidation. Annealing was performed in UHV on a sample with a 30-nm-thick Al film deposited on a glass substrate that had been partially coated with a 20 -nm-thick Ir film. The Ir film was deposited on the glass substrate heated at 540 $\pm 25 \mathrm{~K}$. The reflectance of the two sample areas, i.e., glass/Al and glass/Ir/Al, was monitored after vacuum annealing periods of $1 \mathrm{~h}$ at the increasing temperatures of $370 \pm 25,460 \pm 25$, and $540 \pm 25 \mathrm{~K}$. Figure 3 shows the reflectance in the two sample areas after annealing at 540 $\pm 25 \mathrm{~K}$. The reflectance in the two sample areas decreased after annealing at $540 \pm 25 \mathrm{~K}$ at wavelengths of $73.6 \mathrm{~nm}$ and above. This decrease is attributed to a limited oxidation of $\mathrm{Al}$ in the UHV residual atmosphere of the reflectometer in the period from the deposition to the end of the annealing processes, totaling 7 days. The reflectance of the Ir/Al bilayer remained mostly unchanged below $73.6 \mathrm{~nm}$. To determine whether a hypothetical roughening process of the $\mathrm{Ir}$ 
Larruquert, Méndez, and Aznárez: Nonoxidized Al-overcoated Ir bilayers . . .

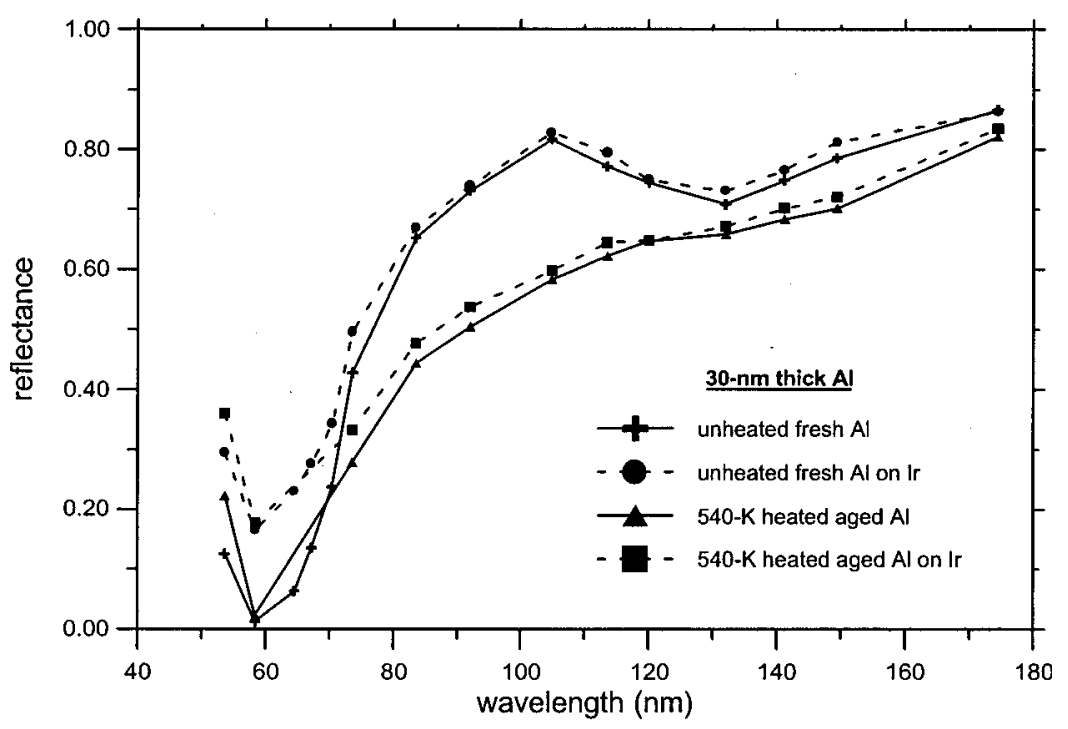

Fig. 3 Reflectance as a function of wavelength for a sample of 30-nm-thick Al film on glass in one area and on a 20-nm-thick Ir film in another area. Reflectance is plotted for the as-deposited sample and for the sample annealed in UHV at $540 \pm 25 \mathrm{~K}$ for $1 \mathrm{~h}$. The sample had been previously annealed at $370 \pm 25$ and $460 \pm 25 \mathrm{~K}$ for periods of $1 \mathrm{~h}$.

or Al films may be observed through specular reflectance measurements, we now estimate the fraction of the radiation scattered by the bilayers that was collected by the detector. The detector, which was placed $200 \mathrm{~mm}$ away from the sample, had an entrance diameter of $8 \mathrm{~mm}$. With this geometry the detector collected only the radiation scattered by roughness structures with spatial wavelengths longer than $5 \mu \mathrm{m}$ for a radiation wavelength of $100 \mathrm{~nm}$. Hence, any roughening of the $\mathrm{Al} /$ vacuum or the $\mathrm{Ir} / \mathrm{Al}$ interface with a structure of spatial range shorter than $5 \mu \mathrm{m}$ would scatter radiation away from the detector, resulting in a reduction of the measured reflectance. The initial stages of a hypothetical reaction/interdiffusion process would be most probably limited to a range shorter than $5 \mu \mathrm{m}$, and the resulting scattered radiation would be placed outside the detector. But even at later stages of the hypothetical reaction there must be short-range roughness components that would scatter radiation outside the detector. Since the reflectance of the annealed Ir/Al bilayer remained mostly unchanged at short wavelengths this shows that the interface Ir/Al was stable upon annealing up to $540 \pm 25 \mathrm{~K}$.

To test the stability of the Ir/Al interface in more detail, a sample with a 135 -nm-thick Al film on a 15 -nm-thick Ir film was prepared. The Ir film was deposited onto a glass substrate at $540 \pm 25 \mathrm{~K}$. Al is a low absorbing material at $58.4 \mathrm{~nm}$ and the $\mathrm{Ir} / \mathrm{Al}$ bilayer presents interference effects in the reflectance-incidence angle plot. Figure 4 shows the reflectance as a function of the angle of incidence measured for the as-deposited bilayer at $58.4 \mathrm{~nm}$. Hunter et al. ${ }^{13}$ performed a similar work on bilayers of $\mathrm{Al}$ on $\mathrm{Au}$ to monitor the progress of the intermetallic diffusion zone. In Fig. 4, several maxima and minima due to interference effects can be observed. If some interdiffusion of $\mathrm{Al}$ and Ir would take place on aging, a change in the low absorbing Al film thickness would result in a different interference pattern. The measured reflectance was modeled as follows. In a first step, the reflectance was calculated assuming smooth interfaces, using the optical constants of Al that are available in the literature ${ }^{14}$ and the optical constants of Ir given in Sec. 3.2. Even though the correct angular positions for the maxima and minima were obtained, the fitting was not quantitatively accurate. By allowing the optical constants of Al to differ from the literature data and by assigning a surface roughness the fitting was improved. In a first ap-

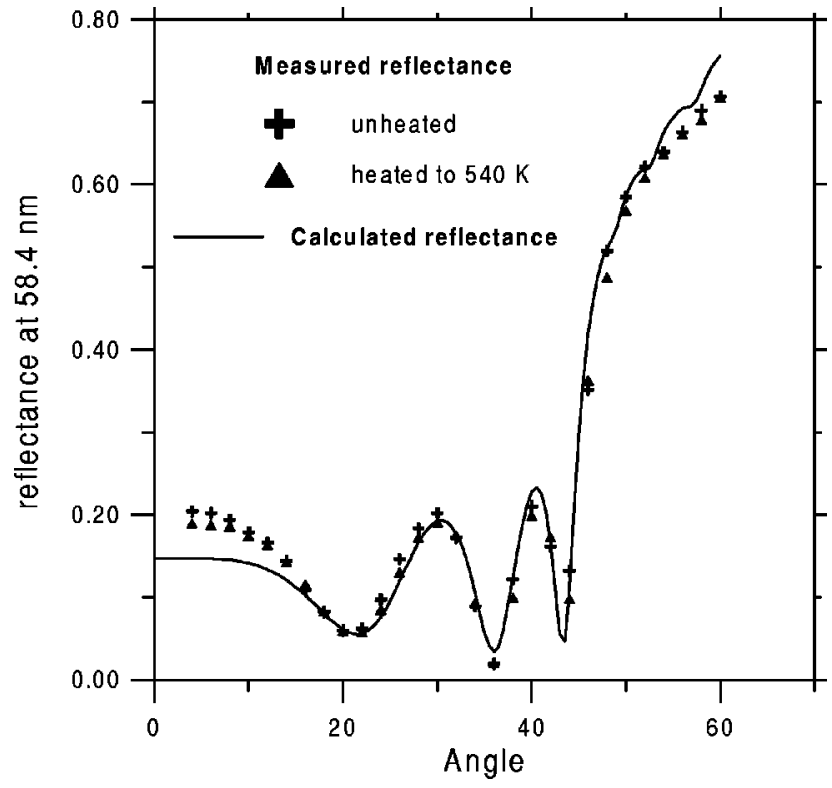

Fig. 4 Reflectance as a function of the incidence angle measured for a 135-nm-thick Al film on a 15-nm-thick Ir film. Reflectance is plotted for the as-deposited sample and for the sample annealed at $540 \pm 25 \mathrm{~K}$ for $1 \mathrm{~h}$. The sample had been previously annealed at $370 \pm 25$ and $460 \pm 25 \mathrm{~K}$ for periods of $1 \mathrm{~h}$. The modeled reflectance is also shown. The angle of incidence is referred to the normal. 


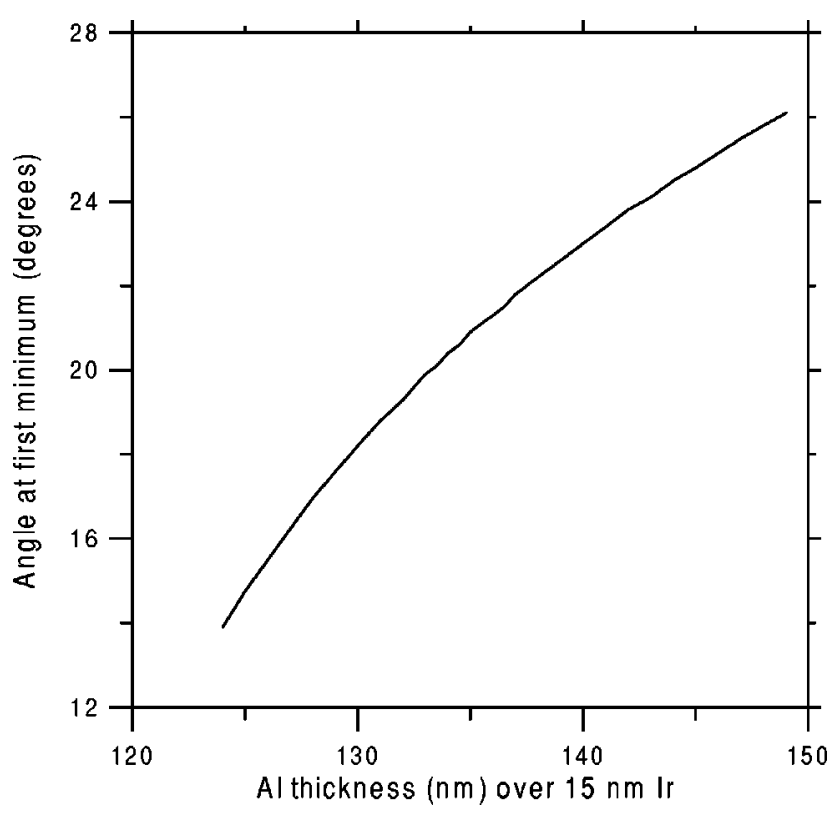

Fig. 5 Calculation of the angle at the first reflectance minimum at $58.4 \mathrm{~nm}$ as a function of thickness of an Al film on a 15-nm-thick Ir film.

proach, the surface roughness was made equal to the substrate roughness. The glass surface roughness had been characterized to have a root mean square (rms) roughness $\sigma=4.9 \mathrm{~nm}$ and an autocorrelation length $T=470 \mathrm{~nm}$, as obtained from angle-resolved scattering on glass substrates coated with an Al film. ${ }^{15}$ The effect of roughness on the specular EUV reflectance was calculated using the vector scattering theory of Croce. ${ }^{16}$ By varying the rms roughness values of the interfaces the fitting was improved. The best results were obtained by assuming $n(\mathrm{Al})=0.717, k(\mathrm{Al})$ $=0.103$, and $\sigma=4.5 \mathrm{~nm}$ for both the Al/vacuum and $\mathrm{Ir} / \mathrm{Al}$ interfaces. The autocorrelation length was maintained at $470 \mathrm{~nm}$. Figure 4 shows the modeled reflectance. The necessity to use the modified optical constants for Al at 58.4 $\mathrm{nm}$ may be understood either because the optical constants of the current films were actually different from the literature data and/or because the simple model used here of two homogeneous films with a roughness described by just two parameters may not be exact.

The sample was successively annealed in vacuum at the increasing temperatures of $370 \pm 25,460 \pm 25$, and 540 $\pm 25 \mathrm{~K}$ for periods of $1 \mathrm{~h}$ and the reflectance of the room temperature sample was measured after every annealing process. The reflectance after the final annealing at 540 $\pm 25 \mathrm{~K}$ is shown in Fig. 4. The reflectance at $58.4 \mathrm{~nm}$ remained mostly unchanged after the three annealing processes, with no change in the interference pattern. A change in the Al film thickness, which would have been caused by the hypothetical diffusion or reaction between $\mathrm{Al}$ and Ir, was simulated through reflectance calculations similar to the preceding. The position of the first minimum at $\sim 21$ deg is very sensitive to the Al thickness. Figure 5 shows a calculation of the dependence of the angle at the first minimum with the Al film thickness. A shift of $\sim 1$ deg per 2-nm thickness change was obtained. No shift of the minimum was observed for the annealed samples, which demon- strates that the $\mathrm{Ir} / \mathrm{Al}$ interface is stable after annealing processes in UHV up to $540 \pm 25 \mathrm{~K}$.

The preceding results contrast with the study performed by Herzig and Spencer, ${ }^{12}$ in which an Ir film deposited on a room-temperature substrate was coated with an Al film of undetermined thickness for Ir film thickness calibration. Within 6 weeks of the overcoating process they observed an important degradation of the coatings that was attributed to the interdiffusion of the metals. There are various important differences in the coating preparation and storage between the work of Herzig and Spencer and ours. Namely, our samples were annealed under UHV with no prior exposure to atmosphere, and our Ir film was deposited on a high-temperature substrate.

We also performed aging tests in atmosphere. The more important aging test for the bilayers would have been to maintain them under UHV for a long enough time compatible with a certain mission, i.e., for years, and then remeasure the reflectance and observe the coatings. That test was not possible in our system. Instead, we stored two samples under normal atmosphere for $5 \mathrm{yr}$ and observed them periodically with a microscope: no signs of degradation were observed for the 5-yr-old samples. Obviously, no EUV reflectance measurements are meaningful for samples that have been stored in atmosphere because of $\mathrm{Al}$ oxidation. This is in disagreement with the result of Herzig and Spencer. ${ }^{12}$ We suggest that this disagreement may be due to the hot temperature of our substrates when Ir was deposited versus the room temperature in their study. The Ir film deposited on a hot substrate results in well-developed crystallites with a minimum of imperfection ${ }^{11}$ and in a more dense material that is expected to be more stable and less prone to interact with other materials such as with $\mathrm{Al}$.

\subsection{Optical Constants of Ir}

The reflectance of Ir as a function of the incidence angle was also measured. These measurements were used to calculate the optical constants of Ir films deposited both on unheated glass substrates as well as on heated glass substrates at $540 \pm 25 \mathrm{~K}$. The search for the optical constants of films was made by the minimization of the following merit function:

$s^{2}=\sum_{i=1, \ldots, m}\{R \exp [\theta(i)]-R[\theta(i), n, k, p]\}^{2}$,

where $R \exp [\theta(i)]$ is the reflectance measured at the angle of incidence $\theta(i)$, and $R[\theta(i), n, k, p]$ is the calculated reflectance for the trial set of optical constants $n$ and $k$ and for the degree of polarization of the beam $p$ that is defined as

$p=\frac{I_{p}-I_{s}}{I_{p}+I_{s}}$,

where $I_{p}$ and $I_{s}$ represent the fraction of the incident intensity with the electric vector parallel and perpendicular, respectively, to the plane of incidence. The number of angles of incidence was $m=9$, namely, $5,15,25,35,45,55,65$, 75 , and $80 \mathrm{deg}$, all of them in the horizontal plane of incidence of the reflectometer. The degree of polarization $p$ of the monochromator was previously obtained from reflec- 
Larruquert, Méndez, and Aznárez: Nonoxidized Al-overcoated Ir bilayers . . .

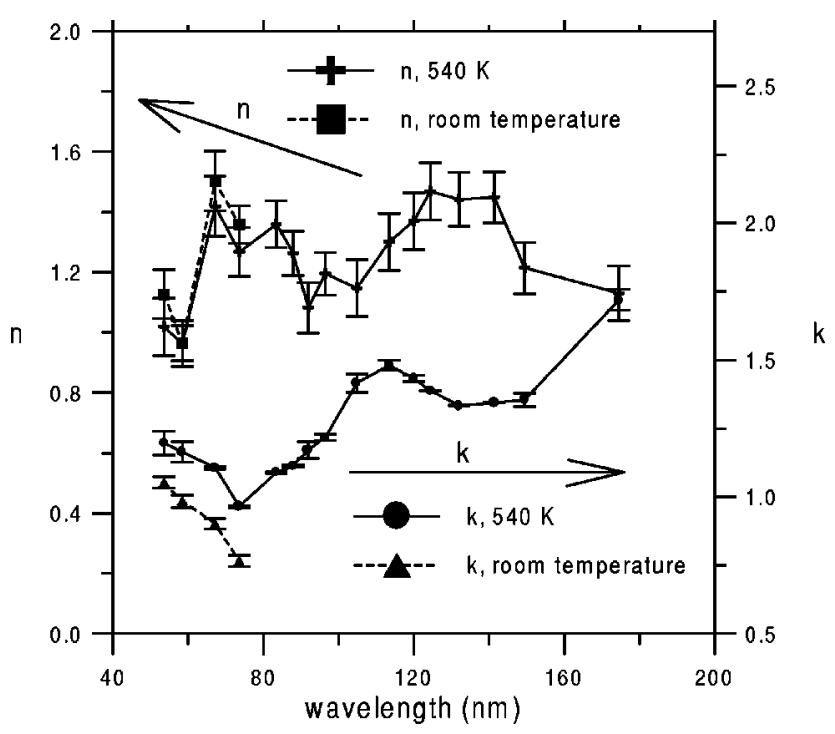

Fig. 6 Optical constants obtained for room-temperature Ir films that were deposited on room temperature as well as on $540 \pm 25 \mathrm{~K}$ hot glass substrate.

tance measurements versus the angle of incidence that were performed in two perpendicular planes of incidence on different samples. The optical constants of glass, which were previously determined from reflectance measurements versus the angle of incidence, were used in the calculation of the optical constants of Ir since the films were not opaque. The optical constants obtained for Ir films are shown in Fig. 6 . The higher reflectance of the films that were deposited on hot substrates corresponds with a higher extinction coefficient $k$, i.e., with a higher absorption.

\section{Summary}

Ir/Al bilayers were prepared by coating an Ir film with an Al film. The Ir film had been evaporated on a heated glass substrate. The bilayers were intended to enhance the reflectance of $\mathrm{Al}$ films below the $\mathrm{Al}$ plasma wavelength $\lambda_{p}(\sim 83$ $\mathrm{nm}$ ), while the high reflectance of Al films above $\lambda_{p}$ is preserved. Bilayers with a 15- to 30-nm-thick Al film deposited on an Ir film resulted in a reflectance increase over the reflectance of single $\mathrm{Al}$ films below $\lambda_{p}$. A higher increase below $\lambda_{p}$ was obtained with a 15-nm-thick Al film, whereas the reflectance above $\lambda_{p}$ was higher for the 30-nmthick $\mathrm{Al}$ film, and the latter was similar to the reflectance of bare Al. Thus, the optimum Al thickness will be a compromise of acceptable reflectance at both spectral regions and will depend on the practical application. The Ir/Al interface was stable after in vacuum sample annealing and after long-term storage under normal atmosphere. Therefore, we conclude that these bilayers may be considered a good choice as coatings for space operating optical instruments applied to EUV astronomy. For this application, the $\mathrm{Al}$ film must be prepared in situ on the orbiting instrument, whereas the Ir film might have been deposited in the laboratory.

\section{Acknowledgments}

We acknowledge Mr. José M. Sánchez Orejuela for his technical assistance. This work was performed under financial support No. ESP1999-1763-E from the National Program for Space Research through Spanish Comisión Interministerial de Ciencia y Tecnología.

\section{References}

1. G. Hass and W. R. Hunter, "Calculated reflectance of aluminumovercoated iridium in the vacuum ultraviolet from $500 \AA$ to $2000 \AA$,' Appl. Opt. 6, 2097-2100 (1967).

2. J. A. Aznárez, J. A. Méndez, J. L. Sacedón, and M. Sánchez-Avedillo, "EDMO: experimental deposition of materials in orbit," Prep. Future 8(3), 12-14 (1998).

3. J. I. Larruquert, J. A. Méndez, and J. A. Aznárez, "Far UV reflectance of UHV prepared Al films and its degradation after exposure to O2," Appl. Opt. 33, 3518-3522 (1994).

4. J. I. Larruquert, J. A. Méndez, and J. A. Aznárez, "Far UV reflectance measurements and optical constants of unoxidized Al films," Appl. Opt. 34, 4892-4899 (1995).

5. J. I. Larruquert, J. A. Méndez, and J. A. Aznárez, "Degradation of far ultraviolet reflectance of aluminum films exposed to atomic oxygen. In-orbit coating application," Opt. Commun. 124, 208-215 (1996).

6. J. I. Larruquert, J. A. Méndez, and J. A. Aznárez, "Optical constants of aluminum films in the extreme ultraviolet interval of $82-77 \mathrm{~nm}$," Appl. Opt. 35, 5692-5697 (1996).

7. J. I. Larruquert, J. A. Méndez, and J. A. Aznárez, "Life prolongation of far ultraviolet reflecting aluminum coatings by periodic recoating of the oxidized surface," Opt. Commun. 135, 60-64 (1997).

8. J. A. Méndez, J. I. Larruquert, and J. A. Aznárez, "Preservation of FUV aluminum reflectance by overcoating with $\mathrm{C}_{60}$ films," Appl. Opt. 39, 149-156 (2000).

9. J. A. Aznánez, J. I. Larruquert, and J. A. Méndez, "Far ultraviolet absolute reflectometer for optical constants determination of ultra high vacuum prepared thin films," Rev. Sci. Instrum. 67, 497-502 (1996).

10. J. I. Larruquert, J. A. Aznárez, and J. A. Méndez, "FUV reflectometer for in situ characterization of thin films deposited under UHV," in Instrumentation for UV/EUV for Astronomy and Solar Missions, S. Fineschi, C. M. Korendyke, O. H. Siegmund, and B. E. Woodgate, Eds., Proc. SPIE 4139, 92-101 (2000).

11. G. Hass, F. G. Jacobus, and W. R. Hunter, "Optical properties of evaporated iridium in the vacuum ultraviolet from $500 \AA$ to $2000 \AA$," J. Opt. Soc. Am. 57, 758-762 (1967).

12. H. Herzig and R. S. Spencer, "Vacuum deposition of iridium on large astronomical mirrors for use in the far UV," Appl. Opt. 21, 15-16 (1982).

13. W. R. Hunter, T. L. Mikes, and G. Hass, "Deterioration of reflecting coatings by intermetallic diffusion," Appl. Opt. 11, 1594-1597 (1972).

14. E. D. Palik, Handbook of Optical Constants of Solids, Academic Press, Orlando, FL (1985)

15. J. I. Larruquert, J. A. Méndez, and J. A. Aznánez, "Empirical relations among scattering, roughness parameters, and thickness of aluminum films," Appl. Opt. 32, 6341-6346 (1993).

16. P. Croce, "Sur l'effet des couches très minces et des rugosités sur la réflexion, la transmission et la diffusion de la lumiére," J. Opt. 8, 127-139 (1977); P. Croce, “Complément à l'étude de l'effet des couches tres minces et des rugosites sur la reflexion, la transmission et la diffusion de la lumiére par un dioptre," J. Opt. 9, 61-63 (1978).

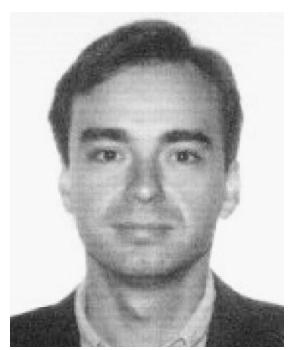

Juan I. Larruquert received his $\mathrm{PhD}$ in physics from Universidad Autónoma de Madrid in 1993. He is a tenured scientist with the Instituto de Física Aplicada of the Spanish National Council for Research (CSIC), where he has been since 1990. He held a National Research Council research associate position with the National Aeronautics and Space Administration (NASA)/ Goddard Space Flight Center from 1996 to 1998. He has been doing research on thin films and multilayer coatings for the far and extreme UV. His main interests are thin films, far to extreme UV, multilayer coatings, space optics, roughness, and scattering. 


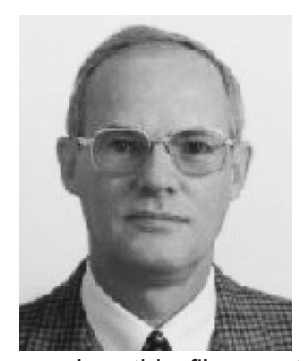

José A. Méndez received his PhD in physics from Universidad de Navarra in 1977. He was an assistant professor with the Universidad del Pais Vasco until 1979 when he joined the Spanish National Council for Research (CSIC), where he has been a senior scientist. He has been doing research on laser speckle, optical and digital image processing, thin films, and multilayer coatings for the far and extreme UV. His research interest areas include image processing, thin films, extreme UV, multilayer coatings, space optics, roughness, and scattering.

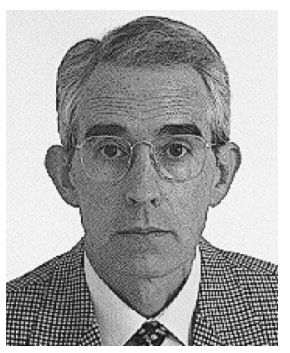

José A. Aznárez is presently a senior research scientist with the Metal Optics Laboratory at the Instituto de Física Aplicada of the Spanish National Council for Research (CSIC). He received his BS in physics in 1963 and his PhD in physics in 1970 from the University of Zaragoza. For more than 20 years he applied electron microscopy techniques to the study of microscopic aggregates on crystals and thin films of photosensitive materials, mainly silver bromide. More recently he has been working on the deposition of thin films under high and ultrahigh vacuum aiming at the preparation of coatings for the far and extreme UV. He was the principal investigator of the scientific team for the European Space Agency (ESA) space experiment EDMO, carried out on the STS-64 shuttle flight in 1994 and devoted to the in-orbit demonstration of thermal evaporation techniques for thin films preparation. 